\title{
A Trellis Coded Modulation Scheme with A Convolutional Processor
}

\author{
Jia-Yin Wang and Mao-Chao Lin \\ Department of Electrical Engineering \\ National Taiwan University \\ Taipei, Taiwan, Republic of China \\ Email address : mclin@cc.ee.ntu.edu.tw
}

\begin{abstract}
We propose a new trellis coded modulation (TCM) scheme. The proposed TCM is encoded by employing the encoder of a convolutional code $C$, which is followed by a convolutional processor and a signal mapper. Large free distances can be easily achieved for the proposed TCM. The decoding can be implemented by using the trellis for the convolutional code $C$ with some feedback information.
\end{abstract}

\section{SUMMARY}

For a proposed TCM, the encoding is shown as Fig. 1. Here

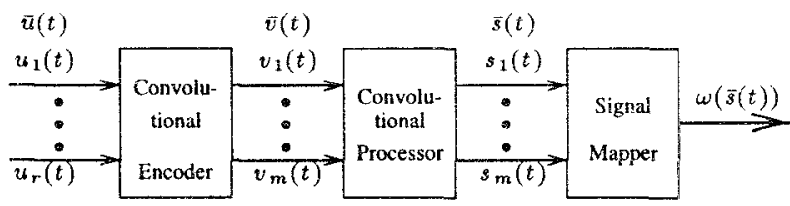

Fig. 1: Encoding structure of the proposed TCM.

$C$ is a rate $r / m$ convolutional code with generator matrix $G_{C}$. In each time unit of encoding, an $r$-bit message $\bar{u}(t)$ is fed into the encoder of $C$, followed by a convolutional processor and a signal mapper to generate a signal point $\omega(\bar{s}(t))$ in the signal space $\Omega$, which may be an 8 PSK signal set, for example. The convolutional processor is the encoder of a rate 1 convolutional code with transfer function matrix $F[1]$.

In the following, we use an example to show how to properly design a convolutional processor to achieve a TCM with large free distance. Define $\Delta_{i}=\min \left\{\| \omega(\bar{s})-\left.\omega\left(\bar{s}^{\prime}\right)\right|^{2}: \bar{s} \neq \bar{s}^{\prime}, s_{j}=\right.$ $s_{j}^{\prime}$ for $\left.j \leq i-1\right\}$. We call $\Delta_{i}$ the level distance of the $i$-th level.

Let $r=2, m=3$ and $\Omega$ be an 8PSK signal set with level distance $\left\{\Delta_{1}, \Delta_{2}, \Delta_{3}\right\}=\{0.586,2,4\}$. Let

$$
F=\left(\begin{array}{ccc}
X^{4 \lambda} & 0 & 0 \\
X^{3 \lambda} & X^{\lambda} & 0 \\
0 & 0 & 1
\end{array}\right)
$$

where $\lambda$ is a positive constant. We have $s_{1}(t)=v_{1}(t-4 \lambda) \oplus$ $v_{2}(t-3 \lambda), s_{2}(t)=v_{2}(t-\lambda)$, and $s_{3}(t)=v_{3}(t)$. Let $V$ denote the sequence $\{\bar{v}(0), \bar{v}(1), \cdots, \bar{v}(t), \cdots\}$ and $d_{i}$ denote $\sum_{t} v_{i}(t)$ for $i=1,2,3$. The squared free distance of the proposed TCM can be shown to be

$$
D_{\text {free }}^{2}=\min _{V \in C, V \neq 0}\left\{d_{1} \cdot \Delta_{1}+d_{2} \cdot\left(\Delta_{2}+\Delta_{1}\right)+d_{3} \cdot \Delta_{3}\right\} .
$$

If we choose $G_{C}=\left(\begin{array}{lll}4 & 4 & 4 \\ 5 & 7 & 0\end{array}\right)$, then $D_{f r e e}^{2}=7.17$. The asymptotic coding gain over uncoded QPSK is $5.54 \mathrm{~dB}$.

The proposed TCM is equivalent to a conventional TCM [2] with a huge number of encoder memory. The trellis for optimum decoding of the proposed TCM will be formidable. However, because of special encoding structure, the proposed TCM can be decoded in a suboptimum way by using the trellis of $C$ (4-state trellis in this examle). The key point of the suboptimum decoding is to determine the branch metric $M_{\bar{v}(t)}(t)$ for $\bar{v}(t)$. With the branch metrics, the Viterbi decoding for the trellis of $C$ is applied, where the truncation length is set to be $\lambda$. The branch metric $M_{\bar{v}(t)}(t)$ is calculated to be the sum of the bit metrics $M_{v_{i}(t)}(t), i=1,2,3$, which can be obtained by using the related received symbols and previously recovered informations. Let $z(t)$ be the received symbol which is the noise-corrupted form of $\omega(s(t))$. In the proposed example, in addition to the recovered information, $M_{v_{3}(t)}$ can be calculated based on $z(t), M_{v_{2}(t)}$ can be calculated based on $z(t+\lambda), z(t+3 \lambda)$, and $M_{v_{1}(t)}$ based on $z(t+4 \lambda), z(t+2 \lambda)$. The overall decoding delay is $5 \lambda-1$ time units. Fig. 2 shows the simulation results. From Fig. 2, we can achieve a coding gain of about $3.5 \mathrm{~dB}$ over uncoded QPSK.

For the aforementioned example, the bit $v_{2}(t)$ is weighted by $\Delta_{2}+\Delta_{1}$. The bit $v_{1}(t)$ and $v_{3}(t)$ is weighted by $\Delta_{1}$ and $\Delta_{3}$ respectively. In general, we may design a transfer function matrix such that the bit $v_{i}(t)$ is weighted by $\Delta_{i}+\ell \Delta_{i-1}$, where $\ell \leq\left\lfloor\Delta_{i} / \Delta_{i-1}\right\rfloor$. The proposed TCM scheme is a generalization of those in [3] and [4].

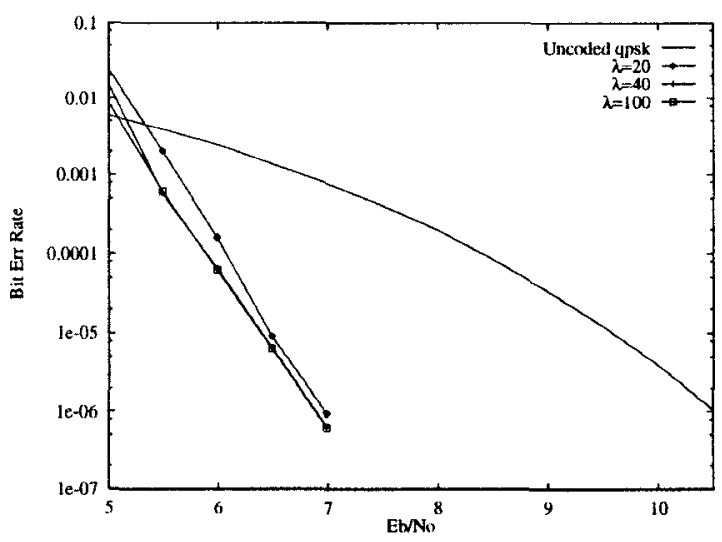

Fig. 2: Simulation results for the proposed example.

\section{REFERENCES}

[1] Shu Lin, Daniel J. Costello, Jr., Error Control Coding : Fundamentals and Applications, Englewood Cliffs: PRENTICEHALL, 1983.

[2] G. Ungerboeck, "Channel coding with multilevel/phase signals," IEEE Trans. on Inform. Theory, vol. IT-28, pp. 55-66, Jan., 1982.

[3] Günter Hellstern, "Coded Modulation with Feedback Decoding Trellis Codes," in Proc. ICC'93 (Geneva, Switzerland, May 1993), pp. 1071-1075.

[4] Jia-Yin Wang and Mao-Chao Lin, "A Multilevel and SingleStage Trellis Coded Modulation Scheme," in Proc. PIMRC'95 (Toronto, Canada, September 1995), pp.1282-1286. 